\title{
Correction: Long-term natural history data in Duchenne muscular dystrophy ambulant patients with mutations amenable to skip exons 44, 45, 51 and 53
}

Claudia Brogna, Giorgia Coratt, Marika Pane, Valeria Ricotti, Sonia Messina, Adele D'Amico, Claudio Bruno, Gianluca Vita, Angela Berardinelli, Elena Mazzone, Francesca Magri, Federica Ricci, Tiziana Mongini, Roberta Battini, Luca Bello, Elena Pegoraro, Giovanni Baranello, Stefano C. Previtali, Luisa Politano, Giacomo P. Comi, Valeria A. Sansone, Alice Donati, Enrico Bertini, Francesco Muntoni, Nathalie Goemans, Eugenio Mercuri, on behalf on the International DMD group

There is an error in affiliation 9 for author Angela Berardinelli. The correct affiliation is: IRCCS Mondino Foundation, Pavia, Italy.

\section{Reference}

1. Brogna C, Coratti G, Pane M, Ricotti V, Messina S, D'Amico A, et al. (2019) Long-term natural history data in Duchenne muscular dystrophy ambulant patients with mutations amenable to skip exons 44,45 , 51 and 53. PLoS ONE 14(6): e0218683. https://doi.org/10.1371/journal.pone.0218683 PMID: 31237898

G

Citation: Brogna C, Coratt G, Pane M, Ricotti V, Messina S, D’Amico A, et al. (2019) Correction: Long-term natural history data in Duchenne muscular dystrophy ambulant patients with mutations amenable to skip exons 44, 45, 51 and 53. PLoS ONE 14(7): e0220714. https://doi.org/ 10.1371/journal.pone.0220714

Published: July 31, 2019

Copyright: ๑ 2019 Brogna et al. This is an open access article distributed under the terms of the Creative Commons Attribution License, which permits unrestricted use, distribution, and reproduction in any medium, provided the original author and source are credited. 